\title{
Analysis of segregated boundary-domain integral equations for mixed variable-coefficient BVPs in exterior domains
}

\author{
O. Chkadua ${ }^{1}$, S.E. Mikhailov ${ }^{2}$, and D. Natroshvili ${ }^{3}$ \\ ${ }^{1}$ A.Razmadze Mathematical Institute, Tbilisi, Georgia; chkadua@rmi.acnet.ge \\ ${ }^{2}$ Brunel University, West London, UK; sergey.mikhailov@brunel.ac.uk \\ ${ }^{3}$ Georgian Technical University, Tbilisi, Georgia; natrosh@hotmail.com
}

\subsection{Introduction}

The direct segregated boundary-domain integral equations for the mixed boundary value problem for a scalar second order elliptic partial differential equation with variable coefficient in an exterior domain in $\mathbb{R}^{3}$ is analysed in this paper. The boundary value problems considered here are well investigated in the literature by the variational methods in the weighted Sobolev spaces, particularly in [Han71, NP73, GN78, Mäu83, Gir87, DL90, Néd01]. For some cases of the PDE with constant coefficients, when the fundamental solution is available, the Dirichlet and Neumann type boundary value problems in exterior domains were also investigated by the classical potential (indirect boundary integral equation) method, see [NP73, GN78, Gir87, DL90, CC00, Néd01] and the references therein.

Our goal here is to show that the mixed problems with variable coefficients can be reduced to some systems of boundary-domain integral equations (BDIEs) and investigate equivalence of the reduction and invertibility of the corresponding boundary-domain integral operators in the weighted Sobolev spaces. To do this, we extend to the exterior domains and weighted spaces the methods developed in [CMN09a] for the interior domains and standard Sobolev (Bessel potential) spaces.

\subsection{Basic notations and spaces}

Let $\Omega=\Omega^{+}$be an unbounded (exterior) open three-dimensional region of $\mathbb{R}^{3}$ such that $\Omega^{-}:=\mathbb{R}^{3} \backslash \bar{\Omega}$ is a bounded open domain. For simplicity, we assume 
that the boundary $\partial \Omega=\partial \Omega^{-}$is a simply connected, closed, infinitely smooth surface.

Let $\rho(x):=\left(1+|x|^{2}\right)^{1 / 2}$ be the weight function and $a \in C^{\infty}\left(\mathbb{R}^{3}\right)$ be such that

$$
\begin{aligned}
0<a_{0}<a(x)<a_{1}<\infty, \\
\quad \rho(x)|\nabla a(x)|+\rho^{2}(x)|\Delta a(x)|<C<\infty, \quad x \in \mathbb{R}^{3} .
\end{aligned}
$$

Let also $\partial_{j}=\partial_{x_{j}}:=\partial / \partial x_{j}(j=1,2,3), \nabla=\partial_{x}=\left(\partial_{x_{1}}, \partial_{x_{2}}, \partial_{x_{3}}\right)$.

We consider below some boundary-domain integral equations associated with the following scalar elliptic differential equation

$$
A u(x):=A\left(x, \partial_{x}\right) u(x):=\sum_{i=1}^{3} \frac{\partial}{\partial x_{i}}\left(a(x) \frac{\partial u(x)}{\partial x_{i}}\right)=f(x), \quad x \in \Omega,
$$

where $u$ is an unknown function and $f$ is a given function in $\Omega$.

In what follows, $H^{s}(\Omega)=H_{2}^{s}(\Omega), H^{s}(\partial \Omega)=H_{2}^{s}(\partial \Omega)$ denote the Bessel potential spaces (coinciding with the Sobolev-Slobodetski spaces if $s \geq 0$ ), $H_{\partial \Omega}^{s}:=\left\{g: g \in H^{s}\left(\mathbb{R}^{3}\right)\right.$, supp $\left.g \subset \partial \Omega\right\}$. For an open set $\Omega$, we, as usual, denote $\mathcal{D}(\Omega)=C_{\text {comp }}^{\infty}(\Omega)$ endowed with sequential continuity, $\mathcal{D}^{*}(\Omega)$ is the Schwartz space of sequentially continuous functionals on $\mathcal{D}(\Omega)$, while $\mathcal{D}(\bar{\Omega})$ is the set of restrictions on $\bar{\Omega}$ of functions from $\mathcal{D}\left(\mathbb{R}^{3}\right)$. We also denote $\widetilde{H}^{s}\left(S_{1}\right)=$ $\left\{g: g \in H^{s}(S)\right.$, supp $\left.g \subset \overline{S_{1}}\right\}, H^{s}\left(S_{1}\right)=\left\{r_{S_{1}} g: g \in H^{s}(S)\right\}$, where $S_{1}$ is a proper submanifold of a closed surface $S$ and $r_{S_{1}}$ is the restriction operator on $S_{1}$.

To make solution of boundary-value problems for (1.2) in infinite domains unique, we will use weighted Sobolev spaces (see e.g. [Han71, NP73, GN78, Mäu83, Gir87, DL90, Néd01]). Let $L_{2}\left(\rho^{-1} ; \Omega\right):=\left\{g: \rho^{-1} g \in L_{2}(\Omega)\right\}$ and $\mathcal{H}^{1}(\Omega)$ be the Beppo-Levi space,

$$
\begin{aligned}
\mathcal{H}^{1}(\Omega):=\left\{g \in L_{2}\left(\rho^{-1} ; \Omega\right): \nabla g\right. & \left.\in L_{2}(\Omega)\right\}, \\
\|g\|_{\mathcal{H}^{1}(\Omega)}^{2} & :=\left\|\rho^{-1} g\right\|_{L_{2}(\Omega)}^{2}+\|\nabla g\|_{L_{2}(\Omega)}^{2} .
\end{aligned}
$$

Using the corresponding property for the space $H^{1}(\Omega)$, it is easy to prove that $\mathcal{D}(\bar{\Omega})$ is dense in $\mathcal{H}^{1}(\Omega)$, cf. [Han71, Theorem I.1], [Gir87, Theorem 2.2]. If $\Omega$ is unbounded, then the seminorm $|g|_{\mathcal{H}^{1}(\Omega)}:=\|\nabla g\|_{L_{2}(\Omega)}$ is equivalent to the norm $\|g\|_{\mathcal{H}^{1}(\Omega)}$ in $\mathcal{H}^{1}(\Omega)$, see e.g. [DL90, Ch. XI, Part B, $\S 1$ ]. If $\Omega$ is bounded, then $\mathcal{H}^{1}(\Omega)=H^{1}(\Omega)$. If $\Omega^{\prime}$ is a bounded subdomain of an unbounded domain $\Omega$ and $g \in \mathcal{H}^{1}(\Omega)$, then $g \in H^{1}\left(\Omega^{\prime}\right)$.

Let us define $\widetilde{\mathcal{H}}^{1}(\Omega)$ as a completion of $\mathcal{D}(\Omega)$ in $\mathcal{H}^{1}\left(\mathbb{R}^{3}\right), \widetilde{\mathcal{H}}^{-1}(\Omega):=$ $\left[\mathcal{H}^{1}(\Omega)\right]^{*}, \mathcal{H}^{-1}(\Omega):=\left[\widetilde{\mathcal{H}}^{1}(\Omega)\right]^{*}, L_{2}(\rho ; \Omega):=\left\{g: \rho g \in L_{2}(\Omega)\right\}$. Evidently $L_{2}(\rho ; \Omega) \subset \mathcal{H}^{-1}(\Omega)$. Any distribution $g \in \widetilde{\mathcal{H}}^{-1}(\Omega)$ has a representation $g=$ $\sum_{i=1}^{3} \partial_{i} g_{i}+g_{0}$, where $g_{i} \in L_{2}(\Omega)$ and $g_{0} \in L_{2}(\rho ; \Omega)$, which implies that $\mathcal{D}(\Omega)$ is dense in $\widetilde{\mathcal{H}}^{-1}(\Omega)$. 
The operator $A$ applied to $u \in \mathcal{H}^{1}(\Omega)$ in the distributional sense is well defined for $a \in L_{\infty}(\Omega)$ as

$$
\langle A u, \varphi\rangle_{\Omega}:=-\langle a \nabla u, \nabla \varphi\rangle_{\Omega}=-\int_{\Omega} a \nabla u \cdot \nabla \varphi d x, \quad \forall u \in \mathcal{H}^{1}(\Omega), \varphi \in \mathcal{D}(\Omega),
$$

where

$$
E(u, \varphi)(x):=a(x) \nabla u(x) \cdot \nabla \varphi(x) .
$$

Thus by density of $\mathcal{D}(\Omega)$ in $\widetilde{\mathcal{H}}^{1}(\Omega)$, we have, $A: \mathcal{H}^{1}(\Omega) \rightarrow \mathcal{H}^{-1}(\Omega)$ is continuous.

From the trace theorem (see, e.g., [LM72]) for $u \in H^{1}(\Omega)$ it follows that if $u \in \mathcal{H}^{1}\left(\Omega^{ \pm}\right)$, then $\gamma^{ \pm} u \in H^{\frac{1}{2}}(\partial \Omega)$, where $\gamma^{ \pm}=\gamma_{\partial \Omega}^{ \pm}$are the trace operators on $\partial \Omega$ from $\Omega^{ \pm}$. We will use $\gamma$ for $\gamma^{ \pm}$if $\gamma^{+}=\gamma^{-}$. We will use also notations $u^{ \pm}$for the traces $\gamma^{ \pm} u$, when this will cause no confusion.

For the linear operator $A$, we introduce the following subspace of $\mathcal{H}^{1}(\Omega)$,

$$
\mathcal{H}^{1,0}(\Omega ; A):=\left\{g \in \mathcal{H}^{1}(\Omega): A g \in L_{2}(\rho ; \Omega)\right\}
$$

endowed with the norm

$$
\|g\|_{\mathcal{H}^{1,0}(\Omega ; A)}^{2}:=\|g\|_{\mathcal{H}^{1}(\Omega)}^{2}+\|\rho A g\|_{L_{2}(\Omega)}^{2},
$$

cf. [GN78].

For $u \in \mathcal{H}^{1}(\Omega)$ (as well as for $u \in H^{1}(\Omega)$ ) the co-normal derivative operators $a \partial_{n} u$ on $\partial \Omega$ may not exist in the classical (trace) sense. However if $u \in \mathcal{H}^{1,0}(\Omega ; A)$, one can correctly define the (generalized) canonical conormal derivative $T^{+} u \in H^{-\frac{1}{2}}(\partial \Omega)$ similar to, for example, [Cos88, Lemma 3.2], [McL00, Lemma 4.3]) as

$$
\left\langle T^{+} u, w\right\rangle_{\partial \Omega}:=\int_{\Omega}\left[\left(\gamma_{-1}^{+} w\right) A u+E\left(u, \gamma_{-1}^{+} w\right)\right] d x \quad \forall w \in H^{\frac{1}{2}}(\partial \Omega),
$$

where $\gamma_{-1}^{+}: H^{\frac{1}{2}}(\partial \Omega) \rightarrow \mathcal{H}^{1}(\Omega)$ is a bounded right inverse to the trace operator $\gamma^{+}: \mathcal{H}^{1}(\Omega) \rightarrow H^{\frac{1}{2}}(\partial \Omega)$. The symbol $\left\langle g_{1}, g_{2}\right\rangle_{\partial \Omega}$ denotes the duality brackets between the spaces $H^{-\frac{1}{2}}(\partial \Omega)$ and $H^{\frac{1}{2}}(\partial \Omega)$, coinciding with $\int_{\partial \Omega} g_{1}(x) g_{2}(x) d S$ if $g_{1}, g_{2} \in L_{2}(\partial \Omega)$. The operator $T^{+}: \mathcal{H}^{1,0}(\Omega ; A) \rightarrow H^{-\frac{1}{2}}(\partial \Omega)$ is continuous and gives the continuous extension on $\mathcal{H}^{1,0}(\Omega ; A)$ of the classical co-normal derivative operator $a \partial_{n}$, where $\partial_{n}=n \cdot \nabla$ and $n=n^{+}$is normal vector on $\partial \Omega$ directed outward the exterior domain $\Omega$.

Similar to the proofs available in [Cos88, Lemma 3.4], [McL00, Lemma 4.3] for $H^{1,0}(\Omega ; A)$ (see also [Mik08, Mik11] for more general spaces $H^{1, t}(\Omega ; A)$ ), one can prove that for $u \in \mathcal{H}^{1,0}(\Omega ; A)$ the first Green identity holds in the form

$$
\left\langle T^{+} u, \gamma^{+} v\right\rangle_{\partial \Omega}=\int_{\Omega}[v A u+E(u, v)] d x \quad \forall v \in \mathcal{H}^{1}(\Omega) .
$$


Then for arbitrary functions $u, v \in \mathcal{H}^{1,0}(\Omega ; A)$ we have the second Green identity,

$$
\int_{\Omega}[v A u-u A v] d x=\left\langle T^{+} u, \gamma^{+} v\right\rangle_{\partial \Omega}-\left\langle T^{+} v, \gamma^{+} u\right\rangle_{\partial \Omega} .
$$

\subsection{Mixed Boundary Value Problem}

The mixed boundary value problem in an exterior domain $\Omega$ is defined as follows.

Find a function $u \in \mathcal{H}^{1,0}(\Omega ; A)$ satisfying the conditions

$$
\begin{aligned}
& A u=f \text { in } \Omega, \\
& r_{\partial \Omega_{D}} \gamma^{+} u=\varphi_{0} \text { on } \partial \Omega_{D}, \\
& r_{\partial \Omega_{N}} T^{+} u=\psi_{0} \text { on } \partial \Omega_{N},
\end{aligned}
$$

where

$$
\varphi_{0} \in H^{\frac{1}{2}}\left(\partial \Omega_{D}\right), \quad \psi_{0} \in H^{-\frac{1}{2}}\left(\partial \Omega_{N}\right), \quad f \in L_{2}(\rho ; \Omega) .
$$

Here $\partial \Omega=\overline{\partial \Omega_{D}} \cup \overline{\partial \Omega_{N}}$, where $\partial \Omega_{D}$ and $\partial \Omega_{N}$ are nonintersecting simply connected submanifolds of $\partial \Omega$ with an infinitely smooth boundary curve $\ell:=$ $\overline{\partial \Omega_{D}} \cap \overline{\partial \Omega_{N}} \in C^{\infty}$.

The first Green identity (1.3) immediately implies the following uniqueness theorem.

Theorem 1. The homogeneous version of BVP (1.5)-(1.7), i.e. with $\varphi_{0}=0$, $\psi_{0}=0, f=0$, has only the trivial solution, while the non-homogeneous problem (1.5)-(1.7) with $\varphi_{0}, \psi_{0}$ and $f$ satisfying (1.8) has at most one solution in $\mathcal{H}^{1,0}(\Omega ; A)$.

Remark 1. Note that the existence of solution in $\mathcal{H}^{1}(\Omega ; A)$ and thus in $\mathcal{H}^{1,0}(\Omega ; A)$ can be proved using the variational setting and the Lax-Milgram theorem, cf. [GN78, Mäu83, Gir87], where this was done for the Dirichlet and Neumann problems for the Poisson equation.

\subsection{Parametrix and Potentials}

It is well known, cf. [Mik02, CMN09a], that the function

$$
P(x, y)=\frac{-1}{4 \pi a(y)|x-y|}, \quad x, y \in \mathbb{R}^{3},
$$

is a parametrix (Levi function) for the operator $A\left(x, \partial_{x}\right)$, i.e.,

$$
A\left(x, \partial_{x}\right) P(x, y)=\delta(x-y)+R(x, y),
$$


where

$$
R(x, y)=\sum_{i=1}^{3} \frac{x_{i}-y_{i}}{4 \pi a(y)|x-y|^{3}} \frac{\partial a(x)}{\partial x_{i}}, \quad x, y \in \mathbb{R}^{3} .
$$

The parametrix $P(x, y)$ is related to a fundamental solution to the operator $A\left(y, \partial_{x}\right):=a(y) \Delta_{x}$ with the "frozen" coefficient $a(x)=a(y)$ and

$$
A\left(y, \partial_{x}\right) P(x, y)=\delta(x-y) .
$$

If $\rho^{-1} \nabla a \in L_{2}(\Omega)$, i.e., $\nabla a \in L_{2}\left(\rho^{-1} ; \Omega\right)$, then for any fixed $y \in \Omega$ and any ball $B_{\epsilon}(y)$ centered at $y$ with sufficiently small radius $\epsilon>0$, we have, $P(., y) \in \mathcal{H}^{1,0}\left(\Omega \backslash B_{\epsilon}(y)\right)$ and $R(., y) \in L_{2}\left(\rho ; \Omega \backslash B_{\epsilon}(y)\right)$. Applying the second Green identity (1.4) in $\Omega \backslash B_{\epsilon}(y)$ with $v=P(y, \cdot)$ and taking usual limits as $\epsilon \rightarrow 0$, cf. [Mir70], we get the third Green identity,

$$
u+\mathcal{R} u-V\left(T^{+} u\right)+W\left(\gamma^{+} u\right)=\mathcal{P} A u \quad \text { in } \Omega
$$

for any $u \in \mathcal{H}^{1,0}(\Omega ; A)$. Here

$$
\mathcal{P} g(y):=\int_{\Omega} P(x, y) g(x) d x, \quad \mathcal{R} g(y):=\int_{\Omega} R(x, y) g(x) d x
$$

are the parametrix-based volume Newton-type and remainder potentials defined for $y \in \mathbb{R}^{3}$, while

$$
V g(y):=-\int_{\partial \Omega} P(x, y) g(x) d S_{x}, \quad W g(y):=-\int_{\partial \Omega}\left[T_{x} P(x, y)\right] g(x) d S_{x}
$$

are surface single layer and double layer potentials, defined for $y \in \mathbb{R}^{3} \backslash \partial \Omega$. The Newton-type and the remainder potential operator given by (1.12) for $\Omega=\mathbb{R}^{3}$ will be denoted as $\mathbf{P}$ and $\mathbf{R}$, respectively. Recall that in the definition of $W$ we assumed $T_{x}=a(x) n(x) \cdot \nabla_{x}$, where $n=n^{+}$is normal vector on $\partial \Omega$ directed outward the exterior domain $\Omega$

From definitions (1.9), (1.10), (1.12)-(1.13) one can obtain representations of the parametrix-based potential operators in terms of their counterparts for $a=1$, i.e. associated with the Laplace operator $\Delta$,

$$
\begin{gathered}
\mathcal{P} g=\frac{1}{a} \mathcal{P}_{\Delta} g, \mathcal{R} g=-\frac{1}{a} \sum_{j=1}^{3} \partial_{j}\left[\mathcal{P}_{\Delta}\left(g \partial_{j} a\right)\right], \\
V g=\frac{1}{a} V_{\Delta} g, W g=\frac{1}{a} W_{\Delta}(a g) .
\end{gathered}
$$

Theorem 2. The following operators are continuous,

$$
\mathbf{P}: \mathcal{H}^{-1}\left(\mathbb{R}^{3}\right) \rightarrow \mathcal{H}^{1}\left(\mathbb{R}^{3}\right),
$$




$$
\begin{aligned}
\mathcal{P} & : \widetilde{\mathcal{H}}^{-1}(\Omega) \rightarrow \mathcal{H}^{1}(\Omega), \\
& : L_{2}(\rho ; \Omega) \rightarrow \mathcal{H}^{1,0}(\Omega ; A), \\
\mathcal{R} & : \mathcal{H}^{1}(\Omega) \rightarrow \mathcal{H}^{1,0}(\Omega ; A), \\
& : L_{2}\left(\rho^{-1} ; \Omega\right) \rightarrow \mathcal{H}^{1}(\Omega), \\
V & : H^{-\frac{1}{2}}(\partial \Omega) \rightarrow \mathcal{H}^{1,0}(\Omega ; A), \\
W & : H^{\frac{1}{2}}(\partial \Omega) \rightarrow \mathcal{H}^{1,0}(\Omega ; A) .
\end{aligned}
$$

Proof. Let $\phi \in \mathcal{D}\left(\mathbb{R}^{3}\right) \subset \mathcal{H}^{-1}\left(\mathbb{R}^{3}\right)$. Then the Newton potential

$$
\mathbf{P}_{\Delta} \phi=\frac{-1}{4 \pi} \int_{\mathbb{R}^{3}} \frac{\phi(x)}{|x-y|} d x
$$

evidently belongs to $\mathcal{H}^{1}\left(\mathbb{R}^{3}\right)$ and solves the Poisson equation $\Delta v=\phi$ in $\mathbb{R}^{3}$. On the other hand, the Laplace operator from $\mathcal{H}^{1}\left(\mathbb{R}^{3}\right)$ to $\mathcal{H}^{-1}\left(\mathbb{R}^{3}\right)$ possesses a continuous inverse operator $\Delta^{-1}: \mathcal{H}^{-1}\left(\mathbb{R}^{3}\right) \rightarrow \mathcal{H}^{1}\left(\mathbb{R}^{3}\right)$, see e.g. [Han71]. This implies

$$
\mathbf{P}_{\Delta} \phi=\Delta^{-1} \phi .
$$

Due to the density of $\mathcal{D}\left(\mathbb{R}^{3}\right)$ in $\mathcal{H}^{-1}\left(\mathbb{R}^{3}\right)$, (1.23) gives a continuous extension of $\mathbf{P}_{\Delta}$ to the operator $\mathcal{H}^{-1}\left(\mathbb{R}^{3}\right) \rightarrow \mathcal{H}^{1}\left(\mathbb{R}^{3}\right)$. The first relation in (1.14) implies (1.16) under condition $\rho|\nabla a|<C$, and (1.17) immediately follows.

To prove (1.18), let us denote by $\tilde{g}$ the extension of a function $g \in L_{2}(\rho ; \Omega)$ by zero outside $\Omega$. Evidently $\tilde{g} \in L_{2}\left(\rho ; \mathbb{R}^{3}\right)$ and $\mathcal{P}_{\Delta} g=\mathbf{P}_{\Delta} \tilde{g} \in \mathcal{H}^{1}\left(\mathbb{R}^{3}\right)$. Taking into account that

$$
A \mathcal{P} g=g-\sum_{j=1}^{3} \partial_{j}\left(\frac{\partial_{j} a}{a} \mathcal{P}_{\Delta} g\right)
$$

conditions (1.1) imply (1.18).

Let us prove the continuity of operator (1.21). For $\phi \in C^{\infty}(\partial \Omega)$ let us consider the single layer potential for the Laplace operator

$$
V_{\Delta} \phi=\frac{1}{4 \pi} \int_{\partial \Omega} \frac{1}{|x-y|} \phi(x) d \Gamma(x)
$$

which evidently belongs to $\mathcal{H}^{1}(\Omega ; \Delta)$ and solves the Dirichlet problem

$$
\Delta v=0 \text { in } \Omega, \quad \gamma^{+} v=w \text { on } \partial \Omega
$$

for $v \in \mathcal{H}^{1}(\Omega ; \Delta)$, where $w=\gamma V_{\Delta} \phi$. By, e.g., [NP73, Lemma 1.1], problem (1.24) is uniquely solvable and its solution is delivered by a continuous operator $Q: H^{\frac{1}{2}}(\partial \Omega) \rightarrow \mathcal{H}^{1}(\Omega ; \Delta)$. Thus

$$
V_{\Delta} \phi=Q \gamma V_{\Delta} \phi
$$

Taking into account the continuity of the operator $\gamma V_{\Delta}: H^{-\frac{1}{2}}(\partial \Omega) \rightarrow$ $H^{\frac{1}{2}}(\partial \Omega)$ and the density of $C^{\infty}(\partial \Omega)$ in $H^{-\frac{1}{2}}(\partial \Omega)$ we arrive at the continuity of $V_{\Delta}: H^{-\frac{1}{2}}(\partial \Omega) \rightarrow \mathcal{H}^{1}(\Omega ; \Delta)$. Then the first relation in (1.15) implies continuity of (1.21) under conditions (1.1). 
Continuity of (1.22) is proved by a similar argument.

Let us prove continuity of (1.19). To this end, let us consider the second relation in (1.14) for a density $\phi \in \mathcal{D}\left(\mathbb{R}^{3}\right)$ and apply the Gauss divergence theorem

$$
\begin{aligned}
\mathcal{R} \phi(y)=\frac{1}{4 \pi a(y)} \sum_{j=1}^{3} \int_{\Omega}\left[\partial_{y_{j}} \frac{1}{|x-y|}\right] \phi(x) \partial_{j} a(x) d x \\
=-\frac{1}{4 \pi a(y)} \sum_{j=1}^{3} \int_{\Omega}\left[\partial_{x_{j}} \frac{1}{|x-y|}\right] \phi(x) \partial_{j} a(x) d x \\
=-\frac{1}{4 \pi a(y)} \sum_{j=1}^{3} \int_{\partial \Omega} \frac{1}{|x-y|}(\gamma \phi(x)) \partial_{n} a(x) d S_{x} \\
\quad+\frac{1}{4 \pi a(y)} \sum_{j=1}^{3} \int_{\Omega} \frac{1}{|x-y|} \partial_{j}\left(\phi(x) \partial_{j} a(x)\right) d x
\end{aligned}
$$

that is,

$$
\mathcal{R} \phi(y)=-V\left[(\gamma \phi) \partial_{n} a\right](y)-\sum_{j=1}^{3} \mathcal{P}\left[\partial_{j}\left(\phi \partial_{j} a\right)\right](y)
$$

Due to the density of $\mathcal{D}\left(\mathbb{R}^{3}\right)$ in $\mathcal{H}^{1}(\Omega)$, the continuity of the operators (1.18) and (1.21) and conditions (1.1), relation (1.25) is valid also for $\phi \in \mathcal{H}^{1}(\Omega)$, thus implying (1.19).

For $\phi \in \mathcal{D}\left(\mathbb{R}^{3}\right)$ the representation similar to (1.25) when $\Omega=\mathbb{R}^{3}$ takes the form

$$
\mathbf{R} \phi(y)=-\sum_{j=1}^{3} \mathbf{P}\left[\partial_{j}\left(\phi \partial_{j} a\right)\right](y) .
$$

Since $\mathcal{D}\left(\mathbb{R}^{3}\right)$ is dense in $L_{2}(\Omega)$, it is evidently dense also in $L_{2}\left(\rho^{-1} ; \mathbb{R}^{3}\right)$. On the other hand, the operator of multiplication with $\partial_{j} a$ is continuous from $L_{2}\left(\rho^{-1} ; \mathbb{R}^{3}\right)$ to $L_{2}\left(\mathbb{R}^{3}\right)$ due to conditions $(1.1)$, while the differential operator $\partial_{j}$ is continuous from $L_{2}\left(\mathbb{R}^{3}\right)$ to $\mathcal{H}^{-1}\left(\mathbb{R}^{3}\right)$. By (1.26) and (1.16) this implies that the operator $\mathbf{R}: L_{2}\left(\rho^{-1} ; \mathbb{R}^{3}\right) \rightarrow \mathcal{H}^{1}\left(\mathbb{R}^{3}\right)$ is continuous. If $g \in L_{2}\left(\rho^{-1} ; \Omega\right)$, then its continuation with zero to the function $\tilde{g} \in L_{2}\left(\rho^{-1} ; \mathbb{R}^{3}\right)$ is a continuous operator and $\mathcal{R} g=\mathbf{R} \tilde{g}$, which implies (1.20).

Let us introduce also the following boundary integral (pseudodifferential) operators of the direct values and of the co-normal derivatives of the single and double layer potentials:

$$
\mathcal{V} g(y):=-\int_{S} P(x, y) g(x) d S_{x}
$$




$$
\begin{aligned}
& \mathcal{W} g(y):=-\int_{S}\left[T\left(x, n(x), \partial_{x}\right) P(x, y)\right] g(x) d S_{x}, \\
& \mathcal{W}^{\prime} g(y):=-\int_{S}\left[T\left(y, n(y), \partial_{y}\right) P(x, y)\right] g(x) d S_{x}, \\
& \mathcal{L}^{ \pm} g(y):=T^{ \pm} W g(y)
\end{aligned}
$$

where $y \in S$.

They can be also presented in terms of their counterparts for $a=1$, i.e. associated with the Laplace operator $\Delta$, see [CMN09a],

$$
\begin{aligned}
& \mathcal{V} g=\frac{1}{a} \mathcal{V}_{\Delta} g, \quad \mathcal{W} g=\frac{1}{a} \mathcal{W}_{\Delta}(a g), \\
& \mathcal{W}^{\prime} g=\mathcal{W}_{\Delta}^{\prime} g+\left[a \frac{\partial}{\partial n}\left(\frac{1}{a}\right)\right] \mathcal{V}_{\Delta} g, \\
& \mathcal{L}^{ \pm} g=\mathcal{L}_{\Delta}(a g)+\left[a \frac{\partial}{\partial n}\left(\frac{1}{a}\right)\right] W_{\Delta}^{ \pm}(a g)
\end{aligned}
$$

where, as usual, the subscript $\Delta$ means that the corresponding surface potentials are constructed by means of the harmonic fundamental solution $P_{\Delta}(x, y)=-(4 \pi|x-y|)^{-1}$. It is taken into account that $a$ and its derivatives are continuous in $\mathbb{R}^{3}$ and

$$
\hat{\mathcal{L}} g:=\mathcal{L}_{\Delta}(a g):=\mathcal{L}_{\Delta}^{+}(a g)=\mathcal{L}_{\Delta}^{-}(a g)
$$

by the Liapunov-Tauber theorem.

The mapping properties of the operators (1.27)-(1.30) are described in details in [CMN09a]. Particularly, their jump relations are given by the following theorem presented in [CMN09a, Theorem 3.3].

Theorem 3. Let $g_{1} \in H^{-\frac{1}{2}}(S)$, and $g_{2} \in H^{\frac{1}{2}}(S)$. Then

$$
\begin{aligned}
& \gamma^{ \pm} V g_{1}(y)=\mathcal{V} g_{1}(y) \\
& \gamma^{ \pm} W g_{2}(y)=\mp \frac{1}{2} g_{2}(y)+\mathcal{W} g_{2}(y), \\
& T^{ \pm} V g_{1}(y)= \pm \frac{1}{2} g_{1}(y)+\mathcal{W}^{\prime} g_{1}(y),
\end{aligned}
$$

where $y \in \partial \Omega$.

Taking trace and co-normal derivative of the third Green identity (1.11) on $\partial \Omega$, we obtain,

$$
\frac{1}{2} \gamma^{+} u+\gamma^{+} \mathcal{R} u-\mathcal{V} T^{+} u+\mathcal{W} \gamma^{+} u=\gamma^{+} \mathcal{P} A u \quad \text { on } \partial \Omega,
$$




$$
\frac{1}{2} T^{+} u+T^{+} \mathcal{R} u-\mathcal{W}_{\partial \Omega}^{\prime} T^{+} u+\mathcal{L}_{\partial \Omega}^{+} \gamma^{+} u=T^{+} \mathcal{P} A u \quad \text { on } \partial \Omega .
$$

For arbitrary functions $u, f, \Psi, \Phi$, let us consider a more general "indirect" integral relation, associated with (1.11),

$$
u(y)+\mathcal{R} u-V \Psi+W \Phi=\mathcal{P} f \quad \text { in } \quad \Omega,
$$

and prove for the weighted spaces the analog of [CMN09a, Lemma 4.1].

Lemma 1. Let $u \in \mathcal{H}^{1}(\Omega), f \in L_{2}(\rho ; \Omega), \Psi \in H^{-\frac{1}{2}}(\partial \Omega), \Phi \in H^{\frac{1}{2}}(\partial \Omega)$ satisfy (1.37). Then $u$ belongs to $\mathcal{H}^{1,0}(\Omega ; A)$ and is a solution of the equation

$$
A u=f \quad \text { in } \Omega
$$

and

$$
V\left(\Psi-T^{+} u\right)(y)-W\left(\Phi-u^{+}\right)(y)=0, y \in \Omega .
$$

Proof. First of all, rewriting (1.37) in the form

$$
u=\mathcal{P} f-\mathcal{R} u+V \Psi-W \Phi,
$$

we conclude by Theorem 2 that $u \in \mathcal{H}^{1,0}(\Omega ; A)$. Thus we can write the third Green identity (1.11) for the function $u$.

Subtracting (1.37) from the identity (1.11), we obtain

$$
-V \Psi^{*}+W \Phi^{*}=\mathcal{P}[A u-f] \text { in } \Omega,
$$

where $\Psi^{*}:=T^{+} u-\Psi, \Phi^{*}:=\gamma^{+} u-\Phi$. Multiplying equality (1.40) by $a(y)$ we get

$$
-V_{\Delta} \Psi^{*}+W_{\Delta}\left(a \Phi^{*}\right)=\mathcal{P}_{\Delta}[A u-f] \quad \text { in } \Omega .
$$

Applying the Laplace operator $\Delta$ to the last equation and taking into consideration that the both functions in the left-hand side are harmonic surface potentials, while the right-hand side function is the classical Newtonian volume potential, we arrive at equation (1.38). Substituting (1.38) back into (1.40) leads to (1.39).

The counterpart of [CMN09a, Lemma 4.2] for unbounded domain $\Omega$ takes the following form.

Lemma 2. (i) Let $\Psi^{*} \in H^{-\frac{1}{2}}(\partial \Omega)$. If

$$
V \Psi^{*}=0 \text { in } \Omega,
$$

then $\Psi^{*}=0$.

(ii) Let $\Phi^{*} \in H^{\frac{1}{2}}(\partial \Omega)$. If 


$$
W \Phi^{*}(y)=0 \text { in } \Omega
$$

then $\Phi^{*}(x)=-C / a(x)$, where $C$ is a constant.

(iii) Let $\partial \Omega=\overline{S_{1}} \cup \overline{S_{2}}$, where $S_{1}$ and $S_{2}$ are nonempty nonintersecting simply connected submanifolds of $\partial \Omega$ with infinitely smooth boundaries. Let $\Psi^{*} \in \widetilde{H}^{-\frac{1}{2}}\left(S_{1}\right), \Phi^{*} \in \widetilde{H}^{\frac{1}{2}}\left(S_{2}\right)$. If

$$
V \Psi^{*}(y)-W \Phi^{*}(y)=0 \text { in } \Omega,
$$

then $\Psi^{*}=0$ and $\Phi^{*}=0$ on $\partial \Omega$.

Proof. The proofs of items (i) and (iii) coincide with the proofs of their counterparts for an interior domain in [CMN09a, Lemma 4.2].

To prove item (ii), we first remark that the Gauss lemma implies that $\Phi_{\Delta}=-C$ satisfies the equation $W_{\Delta} \Phi_{\Delta}=0$ in the exterior domain $\Omega$ for any $C=$ const. Let us check that there is no other solutions of the equation in $\Omega$. By the usual argument, $T^{+} W_{\Delta} \Phi_{\Delta}=T^{-} W_{\Delta} \Phi_{\Delta}=0$ on $\partial \Omega$, which implies $W_{\Delta}=$ const in the interior domain $\Omega^{-}$due to the uniqueness up to a constant of the solution of the Neumann problem in $H^{1}\left(\Omega^{-}\right)$. Then the jump property of $W_{\Delta}$ gives $\Phi_{\Delta}=$ const. Applying the second relation of (1.15) finalizes the proof of item (ii).

\subsection{Segregated BDIEs for the Mixed Problem}

Let $\Phi_{0} \in H^{\frac{1}{2}}(S)$ be an extension of the given function $\varphi_{0}$ in the Dirichlet boundary condition (1.6) from $\partial \Omega_{D}$ to the whole of $\partial \Omega$ and $\Psi_{0} \in H^{-\frac{1}{2}}(S)$ be an extension of the given function $\psi_{0}$ in the Neumann boundary condition (1.7) from $\partial \Omega_{N}$ to the whole of $\partial \Omega$.

We will explore different possibilities of reducing BVP (1.5)-(1.7) to a system of Boundary-Domain Integral Equations (BDIEs) and in all of them we represent in (1.11), (1.35) and (1.36) the trace of the function $u$ and in its co-normal derivative as

$$
\gamma^{+} u=\Phi_{0}+\varphi, \quad \varphi \in \widetilde{H}^{\frac{1}{2}}\left(\partial \Omega_{N}\right) ; \quad T^{+} u=\Psi_{0}+\psi, \quad \psi \in \widetilde{H}^{-\frac{1}{2}}\left(\partial \Omega_{D}\right),
$$

and will regard the new unknown functions $\varphi$ and $\psi$ as formally segregated of $u$. Thus we will look for the triplet

$$
\begin{aligned}
\mathcal{U}=(u, \psi, \varphi)^{\top} & \in \mathbb{H}:=\mathcal{H}^{1,0}(\Omega ; A) \times \widetilde{H}^{-\frac{1}{2}}\left(\partial \Omega_{D}\right) \times \widetilde{H}^{\frac{1}{2}}\left(\partial \Omega_{N}\right) \\
& \subset \mathbb{X}:=\mathcal{H}^{1}(\Omega) \times \widetilde{H}^{-\frac{1}{2}}\left(\partial \Omega_{D}\right) \times \widetilde{H}^{\frac{1}{2}}\left(\partial \Omega_{N}\right) .
\end{aligned}
$$




\section{BDIE system (M11)}

First, using equation (1.11) in $\Omega$, the restriction of equation (1.35) on $\partial \Omega_{D}$, and the restriction of equation (1.36) on $\partial \Omega_{N}$, we arrive at the BDIE system (M11) of three equations for the triplet of unknowns, $(u, \psi, \varphi)$,

$$
\begin{aligned}
u+\mathcal{R} u-V \psi+W \varphi & =F_{0} & & \text { in } \Omega, \\
r_{\partial \Omega_{D}}\left\{\gamma^{+} \mathcal{R} u-\mathcal{V} \psi+\mathcal{W} \varphi\right\} & =r_{\partial \Omega_{D}} \gamma^{+} F_{0}-\varphi_{0} & & \text { on } \partial \Omega_{D}, \\
r_{\partial \Omega_{N}}\left\{T^{+} \mathcal{R} u-\mathcal{W}^{\prime} \psi+\mathcal{L}_{\partial \Omega}^{+} \varphi\right\} & =r_{\partial \Omega_{N}} T^{+} F_{0}-\psi_{0} & & \text { on } \partial \Omega_{N},
\end{aligned}
$$

where

$$
F_{0}:=\mathcal{P} f+V \Psi_{0}-W \Phi_{0} \text { in } \Omega .
$$

We denote the matrix operator of the left hand side of the systems (M11) as

$$
\mathcal{M}^{11}:=\left[\begin{array}{ccc}
I+\mathcal{R} & -V & W \\
r_{\partial \Omega_{D}} \gamma^{+} \mathcal{R} & -r_{\partial \Omega_{D}} \mathcal{V} & r_{\partial \Omega_{D}} \mathcal{W} \\
r_{\partial \Omega_{N}} T^{+} \mathcal{R} & -r_{\partial \Omega_{N}} \mathcal{W}^{\prime} & r_{\partial \Omega_{N}} \mathcal{L}^{+}
\end{array}\right] .
$$

The notation (M11) and the corresponding superscripts mean that system includes the integral operators of the first kind both on the Dirichlet and Neumann parts of the boundary. The other BDIE systems below are also denoted respectively.

\section{BDIE system (M12)}

Here we use equation (1.11) in $\Omega$ and equation (1.35) on the whole of $\partial \Omega$ to arrive at the BDIE system (M12) of two equations for the triplet $(u, \psi, \varphi)$,

$$
\begin{aligned}
u+\mathcal{R} u-V \psi+W \varphi & =F_{0} & & \text { in } \Omega, \\
\frac{1}{2} \varphi+\gamma^{+} \mathcal{R} u-\mathcal{V} \psi+\mathcal{W} \varphi & =\gamma^{+} F_{0}-\Phi_{0} & & \text { on } \partial \Omega .
\end{aligned}
$$

The left hand side matrix operator of the system is

$$
\mathcal{M}^{12}:=\left[\begin{array}{ccc}
I+\mathcal{R} & -V & W \\
\gamma^{+} \mathcal{R} & -\mathcal{V} & \frac{1}{2} I+\mathcal{W}
\end{array}\right] .
$$

\section{BDIE system (M21)}

To arrive at the BDIE system (M21) of two equations for the triplet $(u, \psi, \varphi)$, we use equation (1.11) in $\Omega$ and equation (1.36) on the whole of $\partial \Omega$, 


$$
\begin{aligned}
u+\mathcal{R} u-V \psi+W \varphi & =F_{0} & & \text { in } \Omega, \\
\frac{1}{2} \psi+T^{+} \mathcal{R} u-\mathcal{W}^{\prime} \psi+\mathcal{L}^{+} \varphi & =T^{+} F_{0}-\Psi_{0} & & \text { on } \partial \Omega .
\end{aligned}
$$

The left hand side matrix operator of the system is

$$
\mathcal{M}^{21}:=\left[\begin{array}{ccc}
I+\mathcal{R} & -V & W \\
T^{+} \mathcal{R} & \frac{1}{2} I-\mathcal{W}^{\prime} & \mathcal{L}^{+}
\end{array}\right] .
$$

\section{BDIE system (M22)}

Finally, using equation (1.11) in $\Omega$, the restriction of equation (1.36) on $\partial \Omega_{D}$, and the restriction of equation (1.35) on $\partial \Omega_{N}$, we arrive for the triplet $(u, \psi, \varphi)$ at the BDIE system (M22) of three equations of "almost" the second kind (up to the spaces),

$$
\begin{aligned}
u+\mathcal{R} u-V \psi+W \varphi & =F_{0} & & \text { in } \Omega, \\
\frac{1}{2} \psi+r_{\partial \Omega_{D}}\left\{T^{+} \mathcal{R} u-\mathcal{W}^{\prime} \psi+\mathcal{L}^{+} \varphi\right\} & =r_{\partial \Omega_{D}}\left\{T^{+} F_{0}-\Psi_{0}\right\} & & \text { on } \partial \Omega_{D}, \\
\frac{1}{2} \varphi+r_{\partial \Omega_{N}}\left\{\gamma^{+} \mathcal{R} u-\mathcal{V} \psi+\mathcal{W} \varphi\right\} & =r_{\partial \Omega_{N}}\left\{\gamma^{+} F_{0}-\Phi_{0}\right\} & & \text { on } \partial \Omega_{N} .
\end{aligned}
$$

The matrix operator of the left hand side of the system (M22) takes form

$$
\mathcal{M}^{22}:=\left[\begin{array}{ccc}
I+\mathcal{R} & -V & W \\
r_{\partial \Omega_{D}} T^{+} \mathcal{R} & r_{\partial \Omega_{D}}\left(\frac{1}{2} I-\mathcal{W}^{\prime}\right) & r_{\partial \Omega_{D}} \mathcal{L}^{+} \\
r_{\partial \Omega_{N}} \gamma^{+} \mathcal{R} & -r_{\partial \Omega_{N}} \mathcal{V} & r_{\partial \Omega_{N}}\left(\frac{1}{2} I+\mathcal{W}\right)
\end{array}\right] .
$$

Remark 2. Note that the second relation (1.14) means that if $a=$ const outside a bounded subdomain $\Omega^{\prime} \subset \Omega$, then the operator $\mathcal{R}$ acts only on the restriction $r_{\Omega^{\prime}} u$. This implies that all the BDIE systems reduce in this case to the BDIEs over $\Omega^{\prime}$ and $\partial \Omega$, and are supplemented with the integral representations for $u$ in $\Omega \backslash \bar{\Omega}^{\prime}$ given by the first equations of the systems.

Denoting the right hand sides of the systems (M11), (M12), (M21) and (M22) as $\mathcal{F}^{\alpha \beta}$, the systems can be rewritten as

$$
\mathcal{M}^{\alpha \beta} \mathcal{U}=\mathcal{F}^{\alpha \beta},
$$

where $\alpha, \beta=1,2$. Due to the mapping properties of the potentials, $\mathcal{F}^{\alpha \beta} \in \mathbb{F}^{\alpha \beta}$, while the operators $\mathcal{M}^{\alpha \beta}: \mathbb{H} \rightarrow \mathbb{F}^{\alpha \beta}$ and $\mathcal{M}^{\alpha \beta}: \mathbb{X} \rightarrow \mathbb{Y}^{\alpha \beta}$ are continuous for any $\alpha, \beta=1,2$. Here we denoted 


$$
\begin{aligned}
& \mathbb{F}^{11}:=\mathcal{H}^{1,0}(\Omega ; A) \times H^{\frac{1}{2}}\left(\partial \Omega_{D}\right) \times H^{-\frac{1}{2}}\left(\partial \Omega_{N}\right), \\
& \mathbb{F}^{12}:=\mathcal{H}^{1,0}(\Omega ; A) \times H^{\frac{1}{2}}(\partial \Omega), \\
& \mathbb{F}^{21}:=\mathcal{H}^{1,0}(\Omega ; A) \times H^{-\frac{1}{2}}(\partial \Omega), \\
& \mathbb{F}^{22}:=\mathcal{H}^{1,0}(\Omega ; A) \times H^{-\frac{1}{2}}\left(\partial \Omega_{D}\right) \times H^{\frac{1}{2}}\left(\partial \Omega_{N}\right), \\
& \mathbb{Y}^{11}:=\mathcal{H}^{1}(\Omega) \times H^{\frac{1}{2}}\left(\partial \Omega_{D}\right) \times H^{-\frac{1}{2}}\left(\partial \Omega_{N}\right), \\
& \mathbb{Y}^{12}:=\mathcal{H}^{1}(\Omega) \times H^{\frac{1}{2}}(\partial \Omega), \\
& \mathbb{Y}^{21}:=\mathcal{H}^{1}(\Omega) \times H^{-\frac{1}{2}}(\partial \Omega), \\
& \mathbb{Y}^{22}:=\mathcal{H}^{1}(\Omega) \times H^{-\frac{1}{2}}\left(\partial \Omega_{D}\right) \times H^{\frac{1}{2}}\left(\partial \Omega_{N}\right) .
\end{aligned}
$$

\subsection{BDIE Analysis}

Let us first prove the equivalence theorems.

Theorem 4. Let $\varphi_{0} \in H^{\frac{1}{2}}\left(\partial \Omega_{D}\right), \quad \psi_{0} \in H^{-\frac{1}{2}}\left(\partial \Omega_{N}\right), \quad f \in L_{2}(\rho ; \Omega)$ and let $\Phi_{0} \in H^{\frac{1}{2}}(\partial \Omega)$ and $\Psi_{0} \in H^{-\frac{1}{2}}(\partial \Omega)$ be some extensions of $\varphi_{0}$ and $\psi_{0}$, respectively.

(i) If a function $u \in \mathcal{H}^{1}(\Omega)$ solves the BVP (1.5)-(1.7), then the triplet $(u, \psi, \varphi)$, where

$$
\psi=T^{+} u-\Psi_{0} \in \widetilde{H}^{-\frac{1}{2}}\left(\partial \Omega_{D}\right), \quad \varphi=\gamma^{+} u-\Phi_{0} \in \widetilde{H}^{\frac{1}{2}}\left(\partial \Omega_{N}\right) .
$$

solves the BDIE systems (M11), (M12), (M21), (M22).

(ii) If a triplet $(u, \psi, \varphi) \in \mathcal{H}^{1}(\Omega) \times \widetilde{H}^{-\frac{1}{2}}\left(\partial \Omega_{D}\right) \times \widetilde{H}^{\frac{1}{2}}\left(\partial \Omega_{N}\right)$ solves one of the BDIE systems (M11), (M12) or (M22), then this solution is unique and solves all the systems, including (M21), while $u$ solves the BVP (1.5)-(1.7) and relations (1.44) hold.

Proof. Item (i) immediately follows from the deduction of the BDIE systems (M11), (M12), (M21), (M22).

Using the similarity of Lemma 1 and items (i, iii) of Lemma 2 to their counterparts Lemma 4.1 and Lemma 4.2(i, iii) in [CMN09a] for the bounded domain $\Omega$, the proof of item (ii) of the theorem follows word-for-word the corresponding proofs of Theorems 5.2, 5.5 and 5.12 in [CMN09a].

The situation with uniqueness and equivalence for system (M21) differs from the one for other systems and from its counterpart BDIE system $(\mathcal{T} \mathcal{T})$ in [CMN09a], particularly because item (ii) of Lemma 2 is different from its analog, [CMN09a, Lemma 4.2(ii)]. Thus system (M21) will be further analysed elsewhere.

To prove the invertibility of the counterparts of the operators $\mathcal{M}^{\alpha \beta}$ for bounded domains in [CMN09a], we essentially used there the compactness of the operator $\mathcal{R}: H^{1}(\Omega) \rightarrow H^{1}(\Omega)$ based on the Rellich compactness theorem. However, the latter theorem does not hold for unbounded domains with 
compact boundaries, and to cope with this, we will split the operator $\mathcal{R}$ into two parts, one of which can be made arbitrarily small while the other one is compact.

Lemma 3. Let $\rho(x)|\nabla a(x)| \rightarrow 0$ as $x \rightarrow \infty$. Then for any $\epsilon>0$ the operator $\mathcal{R}$ can be represented as $\mathcal{R}=\mathcal{R}_{c}+\mathcal{R}_{s}$, where $\mathcal{R}_{c}: \mathcal{H}^{1}(\Omega) \rightarrow \mathcal{H}^{1}(\Omega)$ is compact, while $\left\|\mathcal{R}_{s}\right\|_{\mathcal{H}^{1}(\Omega) \rightarrow \mathcal{H}^{1}(\Omega)}<\epsilon$.

Proof. Let $B_{\eta}$ be a ball of radius $\eta$ centered at 0 such $\partial \Omega \subset B_{\eta}$ and let $\mu \in \mathcal{D}\left(\mathbb{R}^{3}\right)$ be a cut-off function such that $\mu=1$ in $B_{\eta}, \mu=0$ in $\mathbb{R}^{3} \backslash B_{2 \eta}$ and $0 \leq \mu(x) \leq 1$ in $\mathbb{R}^{3}$. Denote $\mathcal{R}_{c} g:=\mathcal{R}[\mu g], \mathcal{R}_{s} g:=\mathcal{R}[(1-\mu) g]$.

By (1.25) we have,

$$
\left\|\mathcal{R}_{s} g\right\|_{\mathcal{H}^{1}(\Omega)}=\left\|\sum_{j=1}^{3} \mathcal{P} \partial_{j}\left[(1-\mu) g \partial_{j} a\right]\right\|_{\mathcal{H}^{1}(\Omega)} \leq Q\|\mathcal{P}\|_{\widetilde{\mathcal{H}}^{-1}(\Omega) \rightarrow \mathcal{H}^{1}(\Omega)},
$$

where

$$
\begin{aligned}
Q:=\sum_{j=1}^{3}\left\|\partial_{j}\left[(1-\mu) g \partial_{j} a\right]\right\|_{\widetilde{\mathcal{H}}^{-1}(\Omega)} \leq \sum_{j=1}^{3}\left\|(1-\mu) g \partial_{j} a\right\|_{L_{2}(\Omega)} \\
\quad \leq 3\|g\|_{L_{2}\left(\rho^{-1} ; \Omega\right)}\|\rho \nabla a\|_{L_{\infty}\left(\mathbb{R}^{3} \backslash B_{\eta}\right)} \leq 3\|\rho \nabla a\|_{L_{\infty}\left(\mathbb{R}^{3} \backslash B_{\eta}\right)}\|g\|_{\mathcal{H}^{1}(\Omega)}
\end{aligned}
$$

Thus

$$
\left\|\mathcal{R}_{s}\right\|_{\mathcal{H}^{1}(\Omega) \rightarrow \mathcal{H}^{1}(\Omega)} \leq 3\|\rho \nabla a\|_{L_{\infty}\left(\mathbb{R}^{3} \backslash B_{\eta}\right)}\|\mathcal{P}\|_{\tilde{\mathcal{H}}^{-1}(\Omega) \rightarrow \mathcal{H}^{1}(\Omega)} \rightarrow 0 \text { as } \eta \rightarrow \infty
$$

as claimed.

Let us prove the claim about the operator $\mathcal{R}_{c}$. Since the support of $\mu$ belongs to $\overline{B_{2 \eta}}$, for any fixed $\eta$ the operator $\mathcal{R}_{c}: \mathcal{H}^{1}(\Omega) \rightarrow \mathcal{H}^{1}(\Omega)$ can be represented as $\mathcal{R}_{c} g=\mathcal{R}_{\Omega_{2 \eta}}\left[\mu r_{\Omega_{2 \eta}} g\right]$, where $\Omega_{2 \eta}=\Omega \bigcap B_{2 \eta}$ and the operator $\mathcal{R}_{\Omega_{2 \eta}}$ is given by the second relation (1.12) with $\Omega$ replaced by $\Omega_{2 \eta}$. The operator $\mathcal{R}_{\Omega_{2 \eta}}: L_{2}\left(\Omega_{2 \eta}\right) \rightarrow \mathcal{H}^{1}(\Omega)$ is continuous by (1.20) since $L_{2}\left(\Omega_{2 \eta}\right)=L_{2}\left(\rho^{-1} ; \Omega_{2 \eta}\right)$ for the bounded domain $\Omega_{2 \eta}$. On the other hand, the restriction operator $r_{\Omega_{2 \eta}}: \mathcal{H}^{1}(\Omega) \rightarrow \mathcal{H}^{1}\left(\Omega_{2 \eta}\right)=H^{1}\left(\Omega_{2 \eta}\right)$ is continuous while the imbedding of $H^{1}\left(\Omega_{2 \eta}\right)$ to $L_{2}\left(\Omega_{2 \eta}\right)$ is compact, which implies that the operator $\mathcal{R}_{c}: \mathcal{H}^{1}(\Omega) \rightarrow \mathcal{H}^{1}(\Omega)$ is compact.

Lemma 3 implies the following statement.

Corollary 1. The operator $I+\mathcal{R}: \mathcal{H}^{1}(\Omega) \rightarrow \mathcal{H}^{1}(\Omega)$ is Fredholm with zero index.

Theorem 5. If $\rho(x)|\nabla a(x)| \rightarrow 0$ as $x \rightarrow \infty$, then the following operators are continuous and continuously invertible,

$$
\begin{aligned}
& \mathcal{M}^{11}: \mathbb{X} \rightarrow \mathbb{Y}^{11} \\
& \mathcal{M}^{11}: \mathbb{H} \rightarrow \mathbb{F}^{11} .
\end{aligned}
$$


Proof. Let us consider the operator

$$
\mathcal{M}_{0}^{11}: \mathbb{X} \rightarrow \mathbb{Y}^{11}
$$

where

$$
\mathcal{M}_{0}^{11}:=\left[\begin{array}{ccc}
I & -V & W \\
0 & -r_{\partial \Omega_{D}} \mathcal{V} & r_{\partial \Omega_{D}} \mathcal{W} \\
0 & 0 & r_{\partial \Omega_{N}} \hat{\mathcal{L}}
\end{array}\right],
$$

and $\hat{\mathcal{L}}$ is defined in (1.34). Evidently operator (1.47) is continuous. The diagonal operators of the triangular matrix operator $\mathcal{M}_{0}^{11}$ are continuously invertible (cf. the proof of [CMN09a, Theorem 5.3]), implying that the operator (1.47) is continuously invertible as well.

Let us now represent $\mathcal{R}=\mathcal{R}_{s}+\mathcal{R}_{c}$ by Lemma 3 so that the operator $\mathcal{R}_{s}$ is sufficiently small for the operator

$$
\mathcal{M}_{s}^{11}:=\left[\begin{array}{ccc}
\mathcal{R}_{s} & 0 & 0 \\
r_{\partial \Omega_{D}} \gamma^{+} \mathcal{R}_{s} & 0 & 0 \\
r_{\partial \Omega_{N}} T^{+} \mathcal{R}_{s} & 0 & 0
\end{array}\right]
$$

to satisfy the inequality $\left\|\mathcal{M}_{s}^{11}\right\|_{\mathbb{X} \rightarrow \mathbb{Y}^{11}}<\left\|\left(\mathcal{M}_{0}^{11}\right)^{-1}\right\|_{\mathbb{Y}^{11} \rightarrow \mathbb{X}}$, where $\left(\mathcal{M}_{0}^{11}\right)^{-1}$ is the operator inverse to $\mathcal{M}_{0}^{11}$. Then the operator $M_{0}^{11}+\mathcal{M}_{s}^{11}: \mathbb{X} \rightarrow \mathbb{Y}^{11}$ is continuously invertible, while the operator

$$
\mathcal{M}_{c}^{11}:=\mathcal{M}^{11}-\mathcal{M}_{0}^{11}-\mathcal{M}_{s}^{11}: \mathbb{X} \rightarrow \mathbb{Y}^{11}
$$

is compact by Lemma 3 and by the mapping properties of the operators $\mathcal{W}^{\prime}$ and $\mathcal{L}^{+}-\hat{\mathcal{L}}$, see [CMN09a, Theorems 3.4, 3.6]. This implies that operator $(1.45)$ is a Fredholm operator with zero index. Since by Theorem 4 it is also injective, we conclude that it is invertible.

To prove that the operator (1.46) is also invertible we remark that the unique solution $U \in \mathbb{X}$ of the system $\mathcal{M}^{11} U=\mathcal{F}^{11} \in \mathbb{F}^{11} \subset \mathbb{Y}^{11}$ is delivered by the bounded inverse to the operator (1.45). By equation (1.41) of the system and Lemma 1 we conclude that this solution belongs also to $\mathbb{H}$ and the mapping $\mathbb{F}^{11} \rightarrow \mathbb{H}$ delivered by the inverse to the operator (1.45) is continuous, thus producing the operator inverse to operator (1.46). This completes the proof for the operator $\mathcal{M}^{11}$.

Theorem 6. If $\rho(x)|\nabla a(x)| \rightarrow 0$ as $x \rightarrow \infty$, then the following operators are continuous and continuously invertible,

$$
\begin{aligned}
& \mathcal{M}^{12}: \mathbb{X} \rightarrow \mathbb{Y}^{12} \\
& \mathcal{M}^{12}: \mathbb{H} \rightarrow \mathbb{F}^{12}
\end{aligned}
$$


Proof. To analyse operator $\mathcal{M}^{12}$ let us consider the auxiliary operator

$$
\mathcal{M}_{0}^{12}: \mathbb{X} \rightarrow \mathbb{Y}^{12},
$$

where

$$
\mathcal{M}_{0}^{12}:=\left[\begin{array}{ccc}
I & -V & W \\
0 & -\mathcal{V} & \frac{1}{2} I
\end{array}\right] .
$$

Evidently operator (1.48) is continuous. Any solution $\mathcal{U}=(u, \psi, \varphi)^{\top} \in \mathbb{X}$ of the equation $\mathcal{M}_{0}^{12} \mathcal{U}=\mathcal{F}$, where $\mathcal{F}=\left(\mathcal{F}_{1}, \mathcal{F}_{2}\right)^{\top} \in \mathcal{H}^{1}(\Omega) \times H^{\frac{1}{2}}(\partial \Omega)$ will solve also the following extended system of three equations,

$$
\begin{aligned}
u+W \varphi-V \psi & =\mathcal{F}_{1} \quad \text { in } \quad \Omega, \\
\frac{1}{2} \varphi-\quad \mathcal{V} \psi & =\mathcal{F}_{2} \text { on } \quad \partial \Omega \\
-r_{\partial \Omega_{D}} \mathcal{V} \psi & =r_{\partial \Omega_{D}} \mathcal{F}_{2} \quad \text { on } \partial \Omega_{D},
\end{aligned}
$$

and vice-versa. The diagonal operators of the system,

$$
\begin{aligned}
I & : \mathcal{H}^{1}(\Omega) \rightarrow \mathcal{H}^{1}(\Omega) \\
\frac{1}{2} I & : H^{\frac{1}{2}}(\partial \Omega) \rightarrow H^{\frac{1}{2}}(\partial \Omega), \\
-r_{\partial \Omega_{D}} \mathcal{V} & : \widetilde{H}^{-\frac{1}{2}}\left(\partial \Omega_{D}\right) \rightarrow H^{\frac{1}{2}}\left(\partial \Omega_{D}\right),
\end{aligned}
$$

are continuously invertible implying that the triangular matrix operator of the system mapping $\mathcal{H}^{1}(\Omega) \times H^{-\frac{1}{2}}(\partial \Omega) \times \widetilde{H}^{\frac{1}{2}}\left(\partial \Omega_{N}\right)$ to $\mathcal{H}^{1}(\Omega) \times H^{\frac{1}{2}}(\partial \Omega) \times$ $H^{\frac{1}{2}}\left(\partial \Omega_{D}\right)$ is also invertible. Taking into account that if $\psi \in \widetilde{H}^{-\frac{1}{2}}\left(\partial \Omega_{D}\right)$ solves the third equation of the system, then $\varphi=2\left(\mathcal{F}_{2}+\mathcal{V} \psi\right) \in \widetilde{H}^{\frac{1}{2}}\left(\partial \Omega_{N}\right)$, we arrive at invertibility of the operator (1.48). The rest of the proof coincides wordfor-word with the one for Theorem 5 .

To prove the counterpart of Theorems 5 and 6 for the operator $\mathcal{M}^{22}$, we need the following statement that can be proved similar to [CMN09a, Lemma 5.13 and Corollary 5.14].

Lemma 4. Let $\partial \Omega=\overline{S_{1}} \cup \overline{S_{2}}$, where $S_{1}$ and $S_{2}$ are nonempty nonintersecting simply connected submanifolds of $\partial \Omega$ with infinitely smooth boundaries. For an arbitrary triplet

$$
\mathcal{F}=\left(\mathcal{F}_{1}, \mathcal{F}_{2}, \mathcal{F}_{3}\right)^{\top} \in \mathcal{H}^{1,0}(\Omega ; A) \times H^{-\frac{1}{2}}\left(S_{1}\right) \times H^{\frac{1}{2}}\left(S_{2}\right)
$$

there exists a unique triplet

$$
\left(f_{*}, \Psi_{*}, \Phi_{*}\right)^{\top}=\mathcal{C}_{S_{1}, S_{2}} \mathcal{F} \in L_{2}(\rho ; \Omega) \times H^{-\frac{1}{2}}(\partial \Omega) \times H^{\frac{1}{2}}(\partial \Omega)
$$

such that 


$$
\begin{aligned}
& \mathcal{F}_{1}=\mathcal{P} f_{*}+V \Psi_{*}-W \Phi_{*} \text { in } \Omega^{+}, \\
& \mathcal{F}_{2}=r_{S_{1}} T^{+} \mathcal{F}_{1}-r_{S_{1}} \Psi_{*} \text { on } S_{1}, \\
& \mathcal{F}_{3}=r_{S_{2}} \gamma^{+} \mathcal{F}_{1}-r_{S_{2}} \Phi_{*} \text { on } S_{2} .
\end{aligned}
$$

Moreover, the operator $\quad \mathcal{C}_{S_{1}, S_{2}}: \mathcal{H}^{1,0}(\Omega ; A) \times H^{-\frac{1}{2}}\left(S_{1}\right) \times H^{\frac{1}{2}}\left(S_{2}\right) \rightarrow L_{2}(\Omega ; \rho) \times$ $H^{-\frac{1}{2}}(\partial \Omega) \times H^{\frac{1}{2}}(\partial \Omega)$ is linear and continuous.

Theorem 7. The operator

$$
\mathcal{M}^{22}: \mathbb{H} \rightarrow \mathbb{F}^{22}
$$

is continuous and continuously invertible.

Proof. By Lemma 4 any right hand side $\mathcal{F}=\left(\mathcal{F}_{1}, \mathcal{F}_{2}, \mathcal{F}_{3}\right) \in \mathbb{F}^{22}$ of the equation

$$
\mathcal{M}^{22} \mathcal{U}=\mathcal{F}
$$

can be uniquely represented in form (1.50)-(1.52), where the triplet $\left(f_{*}, \Psi_{*}, \Phi_{*}\right)$ is given by (1.49), $S_{1}=\partial \Omega_{D}, S_{2}=\partial \Omega_{N}$, and the operator $\mathcal{C}_{\partial \Omega_{D}, \partial \Omega_{N}}: \mathbb{F}^{22} \rightarrow$ $L_{2}(\rho ; \Omega) \times H^{-\frac{1}{2}}(\partial \Omega) \times H^{\frac{1}{2}}(\partial \Omega)$ is continuous.

Let us denote by $\mathcal{A}: \mathcal{H}^{1}(\Omega ; A) \rightarrow L_{2}(\rho ; \Omega) \times H^{\frac{1}{2}}\left(\partial \Omega_{D}\right) \times H^{-\frac{1}{2}}\left(\partial \Omega_{N}\right)$ the left hand side operator of the mixed BVP (1.5)-(1.7), which is evidently continuous. By Theorem 1 and Remark 1 (as well as by Theorem 4, e.g. for the system (M11) and Theorem 5), there exists a continuous inverse operator $\mathcal{A}^{-1}: L_{2}(\rho ; \Omega) \times H^{\frac{1}{2}}\left(\partial \Omega_{D}\right) \times H^{-\frac{1}{2}}\left(\partial \Omega_{N}\right) \rightarrow \mathcal{H}^{1,0}(\Omega ; A)$. Then equivalence Theorem 4 for the system (M22) implies that equation (1.54) has a solution $\mathcal{U}=\left(\mathcal{M}^{22}\right)^{-1} \mathcal{F}$, where the operator $\left(\mathcal{M}^{22}\right)^{-1}: \mathbb{F}^{22} \rightarrow \mathbb{H}$ is given by

$$
\begin{aligned}
u & =\mathcal{A}^{-1}\left[\left(\mathcal{C}_{\partial \Omega_{D}, \partial \Omega_{N}} \mathcal{F}\right)_{1}, r_{\partial \Omega_{D}}\left(\mathcal{C}_{\partial \Omega_{D}, \partial \Omega_{N}} \mathcal{F}\right)_{3}, r_{\partial \Omega_{N}}\left(\mathcal{C}_{\partial \Omega_{D}, \partial \Omega_{N}} \mathcal{F}\right)_{2}\right]^{\top}, \\
\psi & =T^{+} u-\left(\mathcal{C}_{\partial \Omega_{D}, \partial \Omega_{N}} \mathcal{F}\right)_{2}, \\
\varphi & =\gamma^{+} u-\left(\mathcal{C}_{\partial \Omega_{D}, \partial \Omega_{N}} \mathcal{F}\right)_{3},
\end{aligned}
$$

and is evidently continuous. Thus the operator $\left(\mathcal{M}^{22}\right)^{-1}$ is the right inverse to the operator (1.53) but due to the injectivity of the latter implied by the equivalence Theorem 4 , the operator $\left(\mathcal{M}^{22}\right)^{-1}$ is the two-side inverse to it.

In the particular case $a=1$ in $\Omega,(1.5)$ becomes the classical Laplace equation, the remainder operator $\mathcal{R}=0$, and the BDIE system (M22) splits into the system of two Boundary Integral Equations (BIEs),

$$
\begin{gathered}
r_{\partial \Omega_{D}}\left(\frac{1}{2} \psi-\mathcal{W}_{\Delta}^{\prime} \psi+\mathcal{L}_{\Delta}^{+} \varphi\right)=r_{\partial \Omega_{D}} T^{+} F_{0}-r_{\partial \Omega_{D}} \Psi_{0} \text { on } \partial \Omega_{D}, \\
r_{\partial \Omega_{N}}\left(\frac{1}{2} \varphi-\mathcal{V}_{\Delta} \psi+\mathcal{W}_{\Delta} \varphi\right)=r_{\partial \Omega_{N}} F_{0}^{+}-r_{\partial \Omega_{N}} \Phi_{0} \text { on } \partial \Omega_{N},
\end{gathered}
$$

and the representation formula for $u$ in terms of $\varphi$ and $\psi$, 


$$
u=F_{0}+V_{\Delta} \psi-W_{\Delta} \varphi \quad \text { in } \Omega .
$$

System (1.55)-(1.56) can be rewritten in the form

$$
\hat{\mathcal{M}}_{\Delta}^{22} \hat{\mathcal{U}}_{\Delta}=\hat{\mathcal{F}}_{\Delta}
$$

where $\hat{\mathcal{U}}_{\Delta}^{\top}:=(\psi, \varphi) \in \widetilde{H}^{-\frac{1}{2}}\left(\partial \Omega_{D}\right) \times \widetilde{H}^{\frac{1}{2}}\left(\partial \Omega_{N}\right)$,

$$
\begin{aligned}
\hat{\mathcal{M}}_{\Delta}^{22} & :=\left[\begin{array}{cc}
r_{\partial \Omega_{D}}\left(\frac{1}{2} I-\mathcal{W}_{\Delta}^{\prime}\right) & r_{\partial \Omega_{D}} \mathcal{L}_{\Delta}^{+} \\
-r_{\partial \Omega_{N}} \mathcal{V}_{\Delta} & r_{\partial \Omega_{N}}\left(\frac{1}{2} I+\mathcal{W}_{\Delta}\right)
\end{array}\right], \\
\hat{\mathcal{F}}_{\Delta}^{22} & :=\left[\begin{array}{c}
r_{\partial \Omega_{D}} T^{+} F_{0}-r_{\partial \Omega_{D}} \Psi_{0} \\
r_{\partial \Omega_{N}} F_{0}^{+}-r_{\partial \Omega_{N}} \Phi_{0}
\end{array}\right] \in H^{-\frac{1}{2}}\left(\partial \Omega_{D}\right) \times H^{\frac{1}{2}}\left(\partial \Omega_{N}\right) .
\end{aligned}
$$

Moreover, the operator $\hat{\mathcal{M}}_{\Delta}^{22}: \widetilde{H}^{-\frac{1}{2}}\left(\partial \Omega_{D}\right) \times \widetilde{H}^{\frac{1}{2}}\left(\partial \Omega_{N}\right) \rightarrow H^{-\frac{1}{2}}\left(\partial \Omega_{D}\right) \times$ $H^{\frac{1}{2}}\left(\partial \Omega_{N}\right)$ is bounded and injective. Similar to [CMN09a, Theorem 5.18], one can prove the following corollary from Theorem 7 .

Theorem 8. The operator $\hat{\mathcal{M}}_{\Delta}^{22}: \widetilde{H}^{-\frac{1}{2}}\left(\partial \Omega_{D}\right) \times \widetilde{H}^{\frac{1}{2}}\left(\partial \Omega_{N}\right) \rightarrow H^{-\frac{1}{2}}\left(\partial \Omega_{D}\right) \times$ $H^{\frac{1}{2}}\left(\partial \Omega_{N}\right)$ is invertible.

Theorem 9. If $\rho(x)|\nabla a(x)| \rightarrow 0$ as $x \rightarrow \infty$, then the operator $\mathcal{M}^{22}: \mathbb{X} \rightarrow \mathbb{Y}^{22}$ is continuous and continuously invertible.

Proof. Let us consider the auxiliary operator

$$
\mathcal{M}_{0}^{22}: \mathbb{X} \rightarrow \mathbb{Y}^{22}
$$

where

$$
\mathcal{M}_{0}^{22}:=\left[\begin{array}{ccc}
I & -V & W \\
0 & r_{\partial \Omega_{D}}\left(\frac{1}{2} I-\mathcal{W}^{\prime}{ }_{\Delta}\right) & r_{\partial \Omega_{D}} \hat{\mathcal{L}} \\
0 & -r_{\partial \Omega_{N}} \mathcal{V} & r_{\partial \Omega_{N}}\left(\frac{1}{2} I+\mathcal{W}\right)
\end{array}\right],
$$

Operator (1.59) is evidently continuous and can be considered as a matrix block-triangle operator with the lower diagonal block

$$
\hat{\mathcal{M}}_{0}^{22}:=\left[\begin{array}{cc}
r_{\partial \Omega_{D}}\left(\frac{1}{2} I-\mathcal{W}^{\prime}{ }_{\Delta}\right) & r_{\partial \Omega_{D}} \hat{\mathcal{L}} \\
-r_{\partial \Omega_{N}} \mathcal{V} & r_{\partial \Omega_{N}}\left(\frac{1}{2} I+\mathcal{W}\right)
\end{array}\right]
$$

Taking into account relations (1.31) and (1.33), we can represent

$$
\hat{\mathcal{M}}_{0}^{22} g=\operatorname{diag}\left(1, \frac{1}{a}\right) \hat{\mathcal{M}}_{\Delta}^{22}[\operatorname{diag}(1, a) g]
$$


where $\operatorname{diag}(1,1 / a)$ and $\operatorname{diag}(1, a)$ are diagonal $2 \times 2$ matrices. The operator $\hat{\mathcal{M}}_{\Delta}^{22}$ given by (1.58) is invertible by Theorem 8 . Since $0<a_{0}<a(x)<a_{1}<$ $\infty$, this implies the invertibility of the operator

$$
\hat{\mathcal{M}}_{0}^{22}: \widetilde{H}^{-\frac{1}{2}}\left(\partial \Omega_{D}\right) \times \widetilde{H}^{\frac{1}{2}}\left(\partial \Omega_{N}\right) \rightarrow H^{-\frac{1}{2}}\left(\partial \Omega_{D}\right) \times H^{\frac{1}{2}}\left(\partial \Omega_{N}\right)
$$

and thus of operator (1.59). The rest of the proof coincides word-for-word with the one for Theorem 5 .

\subsection{Concluding remarks}

Four different segregated direct boundary-domain integral equation systems associated with the mixed (Dirichlet-Neumann) BVP for a scalar "Laplace" PDE with variable coefficient on a three-dimensional unbounded domain have been formulated and analysed in the paper. Equivalence of three of the BDIE systems to the original BVPs was proved in the case when right-hand side of the PDE is from $L_{2}(\rho ; \Omega)$, and the Dirichlet and the Neumann data from the spaces $H^{\frac{1}{2}}\left(\partial \Omega_{D}\right)$ and $H^{-\frac{1}{2}}\left(\partial \Omega_{N}\right)$, respectively. The invertibility of the BDIE operators of these three systems was proved in the corresponding weighted Sobolev spaces.

Using the approach of [Mik06], united direct boundary-domain integrodifferential systems can be also formulated and analysed for the BVPs in exterior domains. The approach can be extended also to more general PDEs and to systems of PDEs, while smoothness of the variable coefficients and the boundary can be essentially relaxed, and the PDE right hand side can be considered in more general spaces, c.f. [Mik05].

Employing methods of [CMN09b], one can consider also the localised counterparts of the BDIEs for BVPs in exterior domains.

Acknowledgement: The work was supported by the grant EP/H020497/1 "Mathematical analysis of localised boundary-domain integral equations for BVPs with variable coefficients" of the EPSRC, UK.

\section{References}

[CC00] Chudinovich, I., Constanda, C.: Variational and Potential Methods in the Theory of Bending of Plates with Transverse Shear Deformation, Chapman \& Hall/CRC, Chapman \& Hall/CRC, Boca Raton - London - New York - Washington, DC (2000).

[CMN09a] Chkadua, O., Mikhailov, S. E., Natroshvili, D.: Analysis of direct boundary-domain integral equations for a mixed BVP with variable coefficient, I: Equivalence and invertibility. Journal of Integral Equations and Applications, 21, 499-543 (2009). 
[CMN09b] Chkadua, O., Mikhailov, S. E., Natroshvili, D.: Analysis of some localized boundary-domain integral equations. Journal of Integral Equations and Applications, 21, 405-445 (2009).

[Cos88] Costabel, M.: Boundary integral operators on Lipschitz domains: elementary results. SIAM J. Math. Anal., 19, 613-626 (1988).

[DL90] Dautray, R., Lions, J.: Mathematical Analysis and Numerical Methods for Science and Technology, volume 4: Integral Equations and Numerical Methods. Springer, Berlin-Heidelberg-New York (1990).

[Gir87] Giroire, J.: Étude de Quelques Problèmes aux Limites Extérieurs et Résolution par Équations Intégrales. Thése de Doctorat d'État, Université Pierre-et-Marie-Curie (Paris-VI) (1987).

[GN78] Giroire, J., Nedelec, J.: Numerical solution of an exterior Neumann problem using a double layer potential. Mathematics of Computation, 32, 973-990 (1978).

[Han71] Hanouzet, B.: Espaces de Sobolev avec poids application au probleme de Dirichlet dans un demi espace. Rend. del Sem. Mat. della Univ. di Padova, XLVI, 227-272 (1971).

[LM72] Lions, J.-L., Magenes, E.: Non-Homogeneous Boundary Value Problems and Applications, volume 1. Springer, Berlin - Heidelberg - New York (1972).

[Mäu83] Mäulen, J.: Lösungen der Poissongleichung und harmonishe Vektorfelder in unbeshränkten Gebieten. Math. Meth. in the Appl. Sci., 5, 233-255 (1983).

[McL00] McLean, W.: Strongly Elliptic Systems and Boundary Integral Equations. Cambridge University Press, Cambridge, UK (2000).

[Mik02] Mikhailov, S. E.: Localized boundary-domain integral formulations for problems with variable coefficients. Engineering Analysis with Boundary Elements, 26, 681-690 (2002).

[Mik05] Mikhailov, S. E.: Analysis of extended boundary-domain integral and integro-differential equations of some variable-coefficient BVP. In: Chen, K. (ed.) Advances in Boundary Integral Methods - Proc. of the 5th UK Conference on Boundary Integral Methods, 106-125, University of Liverpool Publ., Liverpool, UK (2005).

[Mik06] Mikhailov, S. E.: Analysis of united boundary-domain integro-differential and integral equations for a mixed BVP with variable coefficient. Math. Methods in Applied Sciences, 29, 715-739 (2006).

[Mik08] Mikhailov, S. E.: About traces, extensions and co-normal derivative operators on Lipschitz domains. In: Constanda, C., Potapenko, S. (eds.) Integral Methods in Science and Engineering: Techniques and Applications, 149-160, Birkhäuser, Boston (2008).

[Mik11] Mikhailov, S. E.: Traces, extensions and co-normal derivatives for elliptic systems on Lipschitz domains. J. Math. Analysis and Appl., 378, 324-342 (2011).

[Mir70] Miranda, C.: Partial Differential Equations of Elliptic Type. Springer, Berlin - Heidelberg - New York, 2-nd edition (1970).

[Néd01] Nédélec, J.-C.: Acoustic and Electromagnetic Equations. Springer-Verlag, New York (2001).

[NP73] Nedelec, J., Planchard, J.: Une méthode variationelle d'éléments finis pour la résolution numérique d'un probléme extérieur dans $\mathbb{R}^{3}$. RAIRO, 7(R3), 105-129 (1973). 